\title{
Rapid and profound decrease in resorbtion markers preceded early and profound increase in BMD after succesfull surgery in osteitis fibrosa cystica
}

\author{
Daniel Grigorie ${ }^{1,2}$, Andra Caragheorgheopol ${ }^{1}$, Bogdan Stanescu ${ }^{1}$, Dumitru loachim ${ }^{1}$, Maria lacob ${ }^{1}$, Alina Sucaliuc ${ }^{1,2}$ \\ ${ }^{1}$ National Institute of Endocrinology; ${ }^{2}$ "Carol Davila" University of Medicine and Pharmacy, Bucharest, Romania
}

Introduction. Successful parathyroid surgery in patients with OFC leads to early and marked improvements in BMD but data on very early changes in bone markers are missing.

Case report. A 20-yr-old girl was hospitalized because of severe bone pains and multiple pathological fractures (left humerus and left proximal femur) after minimal trauma. She had lost weight, was amenorrheic for the last 12 months and was bleeding from brown tumors in her mouth. She was unable to walk and was complaining for general fatigue, anorexia, polyuria and polydipsia.

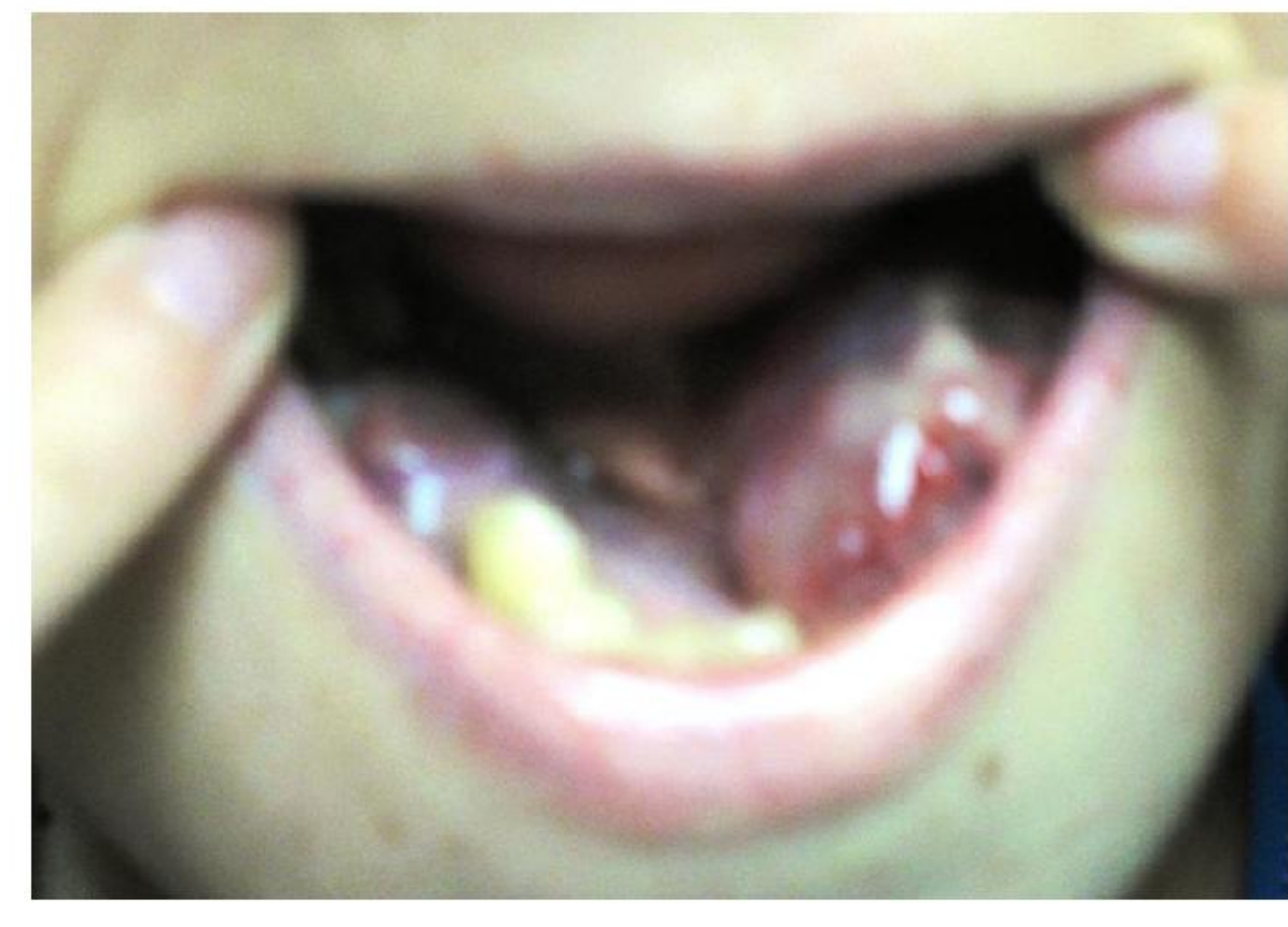

Serum calcium and PTH were of $14.7 \mathrm{mg} / \mathrm{dL}$ and $1243 \mathrm{pg} / \mathrm{mL}$, respectively; alkaline phosphatase activity was $1372 \mathrm{U} / \mathrm{L}$. After hydration with iv sodium chloride, the patient was given iv pamidronate $(60 \mathrm{mg})$ and serum $\mathrm{Ca}$ fell to 12.4 after 2 days. The patient underwent parathyroid surgery and a right superior parathyroid tumor with capsule and blood vessel invasion $(2.2 \mathrm{~g})$ was removed (see figure, $\mathrm{HE}, \mathrm{x} 200$ ). She was treated with oral calcium carbonate ( $2.4 \mathrm{~g}$ daily) and cholecalciferol $2000 \mathrm{U} /$ daily.

A marked reduction in BMD at LS $(0.594 \mathrm{~g} / \mathrm{cm}), F N(0.530 \mathrm{~g} / \mathrm{cm})$ and Total Body $(0.680 \mathrm{~g} / \mathrm{cm})$ was seen by DXA, with Z-scores in the markedly osteoporotic range (-4.9 SD and $-3.9 \mathrm{SD}$, respectively). After 9 mo there were increases of $23.7 \%, 39.6 \%$ and $31.2 \%$, respectively; after 2 yr of $44.1 \%(\mathrm{LS})$ and $64 \%(\mathrm{FN})$.

In the patient and 3 other similar cases (OFC on X-ray) serum CTX decreased after 1 mo by 3-6 times, while OC increased in one patient by 1.2 times and decreased in the other two by $90 \%$ and $53 \%$, respectively. After $12-18$ mo CTX and OC were normal in the three patients with available data. (ref. range for CTX $0.142-0.522 \mathrm{ng} / \mathrm{ml}$, for osteocalcin 8.8-37.6 ng/ml)
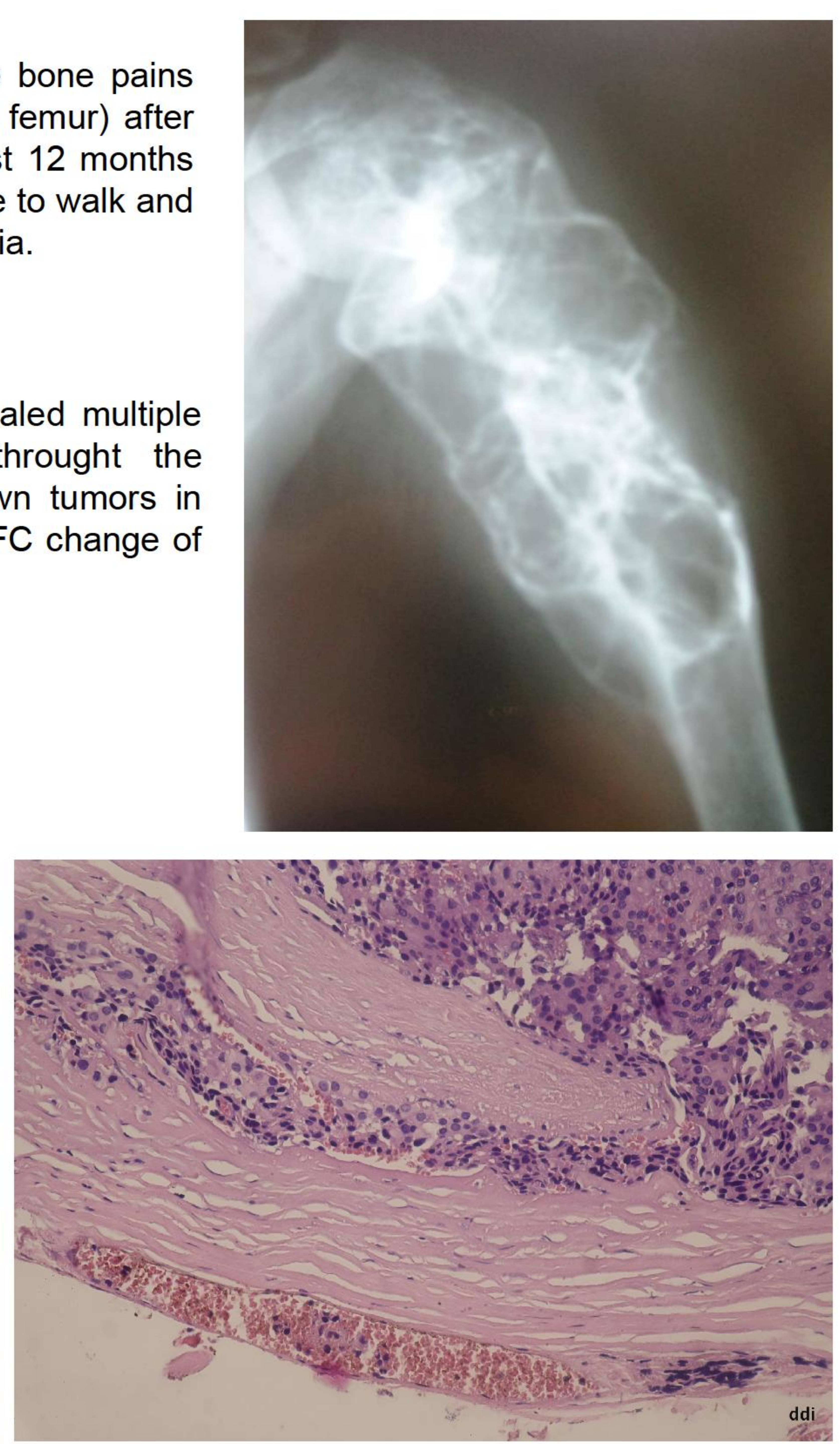

Mean CTX and osteocalcin postoperative changes

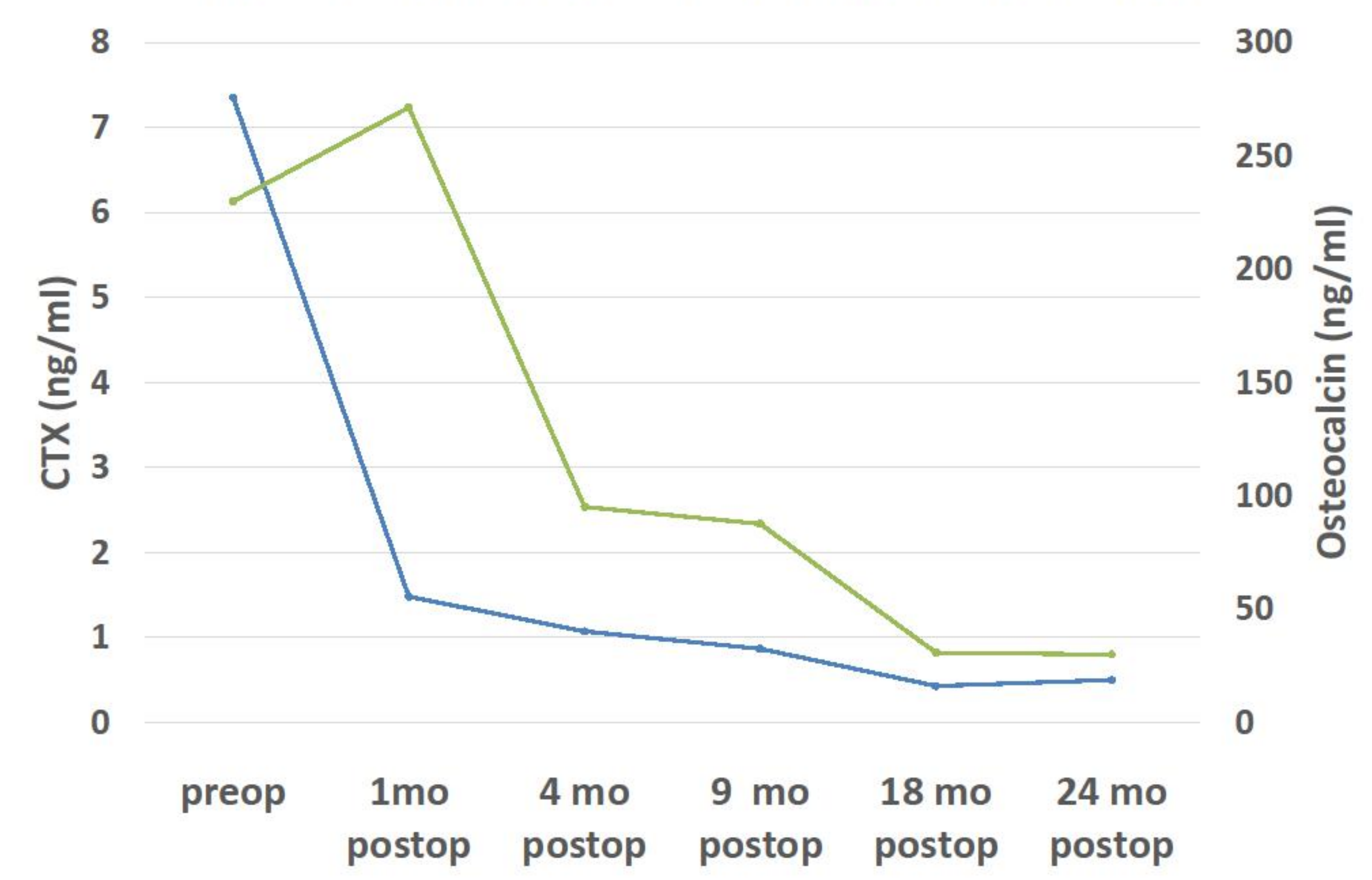

Conclusion: The cases show an unexpected rapid and profound decrease in resorbtion after just one mo after surgery while formation decreased at a much slower pace, changes that preceded the marked increase in BMD, especially at the cortical sites. 